\title{
Qualidade de vida de idosas participantes de um grupo de convivência no município de Bocaiúva - MG
}

\section{Life quality of elderly participating women in cohabitation group in the city of Bocaiúva - MG}

Mariza Alves Barbosa Teles ${ }^{1}$, Mirna Rossi Barbosa Medeiros ${ }^{2}$, Noely Soares Veloso Moura $^{3}$, Cibele Souza Santos ${ }^{4}$, Jucinéia Aparecida Souza ${ }^{5}$, Carlos Roberto Pereira Dias $^{6}$, Tallisson Matheus Oliveira Sales ${ }^{7}$, Raquel Conceição Ferreira ${ }^{8}$

1. ORCID: https://orcid.org/0000-0003-2883-6937. Mestre em Ciências da Saúde. Universidade Estadual de Montes Claros - UNIMONTES, Montes Claros- MG, Brasil. E-mail: aziramteles@gmail.com

2. ORCID: http://orcid.org/0000-0003-4242-4752. Mestre e Doutora em Ciências da Saúde. Faculdades Unidas do Norte de Minas - FUNORTE, Montes Claros-MG, Brasil. E-mail: mirnarossi@hotmail.com

3. ORCID: https://orcid.org/0000-0001-9451-6574. Mestre em Ciências da Saúde. Universidade Estadual de Montes Claros - UNIMONTES, Montes Claros- MG, Brasil. E-mail: noely@gmail.com.br

4. ORCID: https://orcid.org/0000-0002-6632-744X. Enfermeira. Faculdades Unidas do Norte de Minas - FUNORTE, Montes Claros-MG, Brasil. E-mail: enfcibele@gmail.com

5. ORCID: https://orcid.org/0000-0002-1121-0359. Enfermeira. Faculdades Unidas do Norte de Minas - FUNORTE, Montes Claros-MG, Brasil. E-mail: jusenf@gmail.com

6. ORCID: https://orcid.org/0000-0002-9044-0653. Mestre em Desenvolvimento Social. Universidade Estadual de Montes Claros - UNIMONTES, Montes Claros- MG, Brasil. E-mail: professorcarlosunimontes@gmail.com

7. ORCID: https://orcid.org/0000-0003-4150-921X. Enfermeiro. Faculdade de Saúde e Humanidades Ibituruna - FASI, Montes Claros-MG, Brasil. E-mail: matheustallisson@gmail. com.

8. ORCID: https://orcid.org/0000-0001-8897-9345. Doutora em Odontologia. Faculdade de Odontologia da Universidade Federal de Minas Gerais - UFMG, Belo Horizonte - MG Brasil. E-mail: raqueldont@gmail.com

CONTATO: Autor correspondente: Tallisson Matheus Oliveira Sales | Endereço: AV. Profa Aida Mainartina Paraíso, 99- Ibituruna Montes Claros-MG, 39408-007. Telefone: (38) 99136-5574: E-mail: matheustallisson@gmail.com 
RESUMO Qualidade de vida é um conceito multidimensional e complexo, que refere à percepção do indivíduo sobre a condição de sua vida. $O$ objetivo deste estudo foi avaliar a qualidade de vida de idosas participantes de um grupo de convivência. A amostra foi composta de 110 mulheres com idade a partir de 60 anos. Trata-se de um estudo transversal, analítico realizado no município de Bocaiúva- MG. Para a coleta dos dados foram utilizados os instrumentos WHOQOL-OLD, WHOQOL-bref e um questionário sociodemográfico. Os resultados demonstram que alguns domínios exercem maior influência na qualidade de vida em mulheres idosas, que obtiveram maiores scores no domínio geral em qualidade de vida 67,84 (DP $\pm 18,11)$ e domínio psicológico $67,45$ (DP $\pm 9,86)$. A qualidade de vida é um importante indicador de saúde, e os questionários são instrumentos preciosos que podem avaliá-la, e ainda dar subsídios para tomadas de ações que visem promover o bem-estar da população idosa.

DESCRITORES: Qualidade de Vida. Feminização. Envelhecimento.

\begin{abstract}
Life quality is a multidimensional and complex concept, which refers to the individual's perception of their life condition. The aim of this study was to assess the life quality of elderly women participating in a social group. The sample consisted of 110 women aged over 60 years. This is a cross-sectional, analytical study carried out in the city of Bocaiúva-MG. For data collection, the instruments WHOQOL-OLD, WHOQOL-bref and a sociodemographic questionnaire were used. The results demonstrate that some domains exert greater influence on the life quality of elderly women, that obtained higher scores in the general domain of life quality $67.84(S D \pm 18.11)$ and psychological domain 67.45 (SD \pm 9.86 ). Life quality is an important health indicator, and questionnaires are precious instruments that can assess it, and also provide subsidies for taking actions aimed at promoting the well-being of the elderly population.
\end{abstract}

DESCRIPTORS: Life quality. Feminization. Aging. 


\section{INTRODUÇÃO}

O envelhecimento da população por si só não é suficiente se não houver o aumento da qualidade de vida associado. Esse fenômeno de extensão da vida ocorreu primeiro nos países desenvolvidos, mas, recentemente, nos países em desenvolvimento, o envelhecimento da população tornou-se relevante. No Brasil, o número de idosos ( $\geq 60$ anos) aumentou de 3 milhões em 1960 para 7 milhões em 1975 e 14 milhões em 2002 (um aumento de $500 \%$ nos últimos 40 anos), e deve chegar a 32 milhões em 2020. Por exemplo, em um país como a Bélgica, demorou cem anos para o tamanho da população idosa dobrar. O resultado dessa dinâmica é a crescente demanda por serviços de saúde.

Essa rápida transição demográfica, não deve ser considerada como um problema, mas sim como uma alteração demográfica que demanda estudos e ações que possibilitem uma inclusão da população idosa, gerando dignidade e bem-estar a quem envelhece ${ }^{2}$.

A Organização Mundial de Saúde (OMS) considera idoso o indivíduo que tenha idade superior a 60 anos, ao passo que a Organização das Nações Unidas (ONU) aceita como ponto de corte 65 anos de idade para os países desenvolvidos. No Brasil, a Lei $n^{\circ}$. 8.842, que dispõe sobre a Política Nacional do Idoso, e o Estatuto do Idoso, consideram idosa a pessoa com idade maior ou igual há 60 anos $^{3}$. A maioria da população idosa no Brasil é constituída por mulheres, e com isso, alguns estudos têm se voltado para as implicações do envelhecimento nesse gênero ${ }^{4}$.

O envelhecimento produz efeitos físicos e emocionais diferenciados de uma pessoa para outra, em decorrência de fatores diversificados, visto que a limitação permeia a cotidianidade do indivíduo, bem como o contexto em que vive, levando-se em consideração a alimentação, condicionamento físico, econômico, os quais contribuem para que se tenha um envelhecimento com qualidade e menos risco de enfermidade 5 . As mulheres possuem características que as tornam mais vulneráveis, como maior longevidade, embora mais suscetíveis a doenças; maior probabilidade de problemas relacionados a adaptações à senescência, dentre outras alterações ${ }^{4}$.

As chances de mulheres ficarem viúvas aumentam com a idade, e as possibilidades destas se casarem novamente são muito reduzidas, já que hoje, a maioria de novos casamentos de homens idosos acontece com mulheres mais jovens, e o mesmo não acontece com as mulheres idosas ${ }^{6}$. Dentre alguns fatores, não ter companheiro pode estar relacionado a baixos níveis de qualidade de vida ${ }^{7}$. Atualmente, conseguir uma sobrevida cada vez maior não é o único grande desafio, mas também a oportunidade de desfrutarem desses anos adicionais com dignidade e com qualidade de vida ${ }^{8}$.

O termo qualidade de vida abrange muitos conceitos, mas neste estudo será adotado o da Organização Mundial de Saúde (OMS) que desenvolveu uma definição trans- 
cultural, considerada subjetiva, multidimensional, com aspectos positivos e negativos, que é a percepção do indivíduo de sua posição na vida, no contexto da cultura e no sistema de valores nos quais ele vive e em relação aos seus objetivos, expectativas, padrões e preocupações ${ }^{9}$.

A qualidade de vida engloba um conceito amplo de bem-estar, mas depende do autojulgamento do próprio indivíduo, o quanto ele está ou não satisfeito com a qualidade subjetiva de sua vida. É um conceito abstrato que depende de padrões históricos, culturais, sociais e até mesmo individuais. A avaliação da qualidade de vida de determinado indivíduo varia em função das três dimensões nas quais o sujeito se encontra inserido, que é a física, a psicológica e a social ${ }^{10}$. Uma qualidade de vida boa ou excelente é aquela que oferece um mínimo de condições para que os indivíduos possam desenvolver o máximo de suas potencialidades, vivendo, sentindo ou amando, trabalhando, produzindo bens e serviços, ou simplesmente existindo ${ }^{11}$.

Para avaliação da qualidade de vida da população idosa, foi desenvolvido pela World Health Organization Quality of Life Group (Grupo WHOQOL) um instrumento de análise ${ }^{11}$. $O$ instrumento permite a avaliação do impacto da prestação do serviço e de diferentes estruturas de atendimento social e de saúde sobre a qualidade de vida de idosos. Viabiliza especialmente a identificação das possíveis consequências das políticas sobre qualidade de vida para essa população e uma compreensão mais clara das áreas de investimento para se obter melhores ganhos na qualidade de vida ${ }^{12}$. Nesse contexto, destaca-se a relevância de se investigar as condições que interferem no bem-estar na velhice.

Os grupos de terceira idade têm se mostrado muito eficiente, no intuito de criarem alternativas de intervenção e de se proporem ações e políticas na área da saúde para atender às demandas da população $0^{8,13}$.

Os grupos de convivência são locais que passam a representar para os idosos um novo espaço de expressão, aprendizagem, troca de experiências, valorização pessoal, social e de desenvolvimento biopsicossociocultural, tornando assim, um projeto de caráter preventivo, terapêutico e promocional, que visa criar oportunidades para que os idosos possam melhorar e exercitar a convivência grupal, cooperação, participação cidadã e consequente organização, influindo diretamente em sua qualidade de vida ${ }^{14}$. A inserção de idosos em grupos de suporte social proporciona uma mudança no paradigma de velhice enquanto limitação e incapacidade, porque nesses grupos é possível encontrar idosos ativos, autônomos, satisfeitos com sua condição geral e que se relacionam interpessoalmente com outras pessoas de mesma faixa etária ou não ${ }^{10}$.

Os grupos de convivência trazem um significado de grande importância na vida das pessoas idosas através do convívio e do afloramento de várias demandas, como a busca 
de direitos sociais e de cidadania, combate ao analfabetismo, promoção de atividades de lazer (bailes, bingos, viagens, encontros, gincanas, torneios, dentre outros), sobre o processo de envelhecimento e político envolvendo o cidadão e, principalmente, sobre o Estatuto do Idoso. Esses grupos possibilitam aos idosos participar e expressar suas dúvidas e necessidades pessoais, capacitando-os a tomarem decisões, refletirem e exporem seus pensamentos, transformando em certa medida suas vidas ${ }^{14}$.

Nesse contexto, o objetivo deste estudo foi avaliar a qualidade de vida de mulheres idosas pertencentes ao grupo de convivência do município de Bocaiúva-MG/Brasil, e compará-la entre aquelas que possuem ou não companheiro.

\section{MÉTODO}

Trata-se de um estudo quantitativo, analítico do tipo transversal, realizado no mês de outubro de 2012, em um grupo de convivência coordenado pela Secretaria Municipal de Promoção e Assistência Social no município de Bocaiuva, Minas Gerais.

O grupo é formado por dois núcleos: um no Bairro Bonfim, composto por 73 participantes, e outro no Bairro Pernambuco, constituído por 138 idosas. As atividades realizadas são as mesmas em ambos os núcleos. Para a coleta de dados, foi realizado um contato prévio com a coordenadora geral do projeto e com a Secretaria Municipal de Promoção e Assistência Social, tendo sido agendadas as visitas ao grupo. Foram feitos oito encontros para cada núcleo, que ocorreram em horários habituais de participação das usuárias, sendo as atividades rotineiras dos grupos não interrompidas por conta da coleta de dados da pesquisa.

Foram incluídas as participantes com idade igual ou superior a 60 anos, sendo todas do sexo; que frequentavam o projeto há pelo menos dois meses. Aquelas que apresentaram dificuldades de compreensão para responder aos instrumentos, que não estiveram presentes em pelo menos um dos oito encontros feitos para a coleta de dados e que não aceitaram a participar voluntariamente da pesquisa, mediante a assinatura do Termo de Consentimento Livre e Esclarecido, foram excluídas. Desta forma, fizeram parte desta pesquisa 110 idosas, que foram convidadas a participar do estudo de forma voluntária, momentos antes de se iniciarem as atividades do referido grupo de convivência. Sendo aceito o convite, foram encaminhadas até uma sala reservada para a aplicação dos questionários.

Foi criado pelas autoras um instrumento específico para a coleta de dados deste estudo, caracterizando os aspectos: sexo, idade, ocupação, estado civil, religião, renda, escolaridade, ter ou não plano de saúde e arranjo familiar (com quem a idosa convivia). Também foi utilizado o WHOQOL-OLD, instrumento desenvolvido pela Organização Mundial da Saúde, e validado no Brasil, especificamente, para a população idosa ${ }^{15}$. 
O questionário WHOQOL-OLD é composto por 24 itens divididos em seis facetas ${ }^{15}$. Cada uma engloba quatro itens, portanto, para todas as facetas o escore dos valores pode variar de quatro a 20. Os escores destas seis facetas ou os valores dos 24 itens do módulo WHOQOL-OLD podem ser combinados para produzir um escore geral ("global") para a qualidade de vida em adultos idosos. Escores altos representam uma alta qualidade de vida e escores baixos representam uma baixa qualidade de vida ${ }^{12}$.

Para avaliar a qualidade de vida, também foi utilizado o instrumento WHOQOL-bref, validado no Brasil ${ }^{16}$, que é uma versão reduzida do questionário completo original com 100 questões (WHOQOL-100). O WHOQOL-bref é composto por 26 questões, sendo duas delas gerais e 24 representantes de cada uma das 24 facetas que compõem o instrumento original. Compreende quatro domínios da qualidade de vida, sendo que cada um tem por objetivo analisar, respectivamente: a capacidade física, o bem-estar psicológico, as relações sociais e o meio-ambiente onde o indivíduo está inserido.

As questões do instrumento do WHOQOL-bref contêm questões com opções de respostas em escala de Likert, que mede intensidade (nada a extremamente), capacidade (nada a completamente), frequência (nunca a sempre) e avaliação (muito satisfeito a muito insatisfeito e muito ruim a muito bom). Conforme orientações da Organização Mundial de Saúde os escores de cada domínio do WHOQOL-bref foram transformados em escalas de 0 a 100, e quanto maior o escore no questionário, melhor é a qualidade de vida ${ }^{12}$.

Os dados foram tabulados empregando o Programa Epidata versão $3.1 \mathrm{~b}$, e para análise foi utilizado o pacote estatístico SPSS 15.0. Foram obtidas as médias e desvios-padrão em cada item dos instrumentos utilizados e feita comparação das facetas e domínios da qualidade de vida entre quem possui e não possui companheiro, por meio da Razão de Prevalência (RP) e do teste não paramétrico de Mann-Whitney, com valor significativo de $p<0,05$.

Este estudo foi submetido ao Comitê de Ética em Pesquisa com Seres Humanos das Faculdades Integradas do Norte de Minas - SOEBRAS e, após avaliação, foi devidamente aprovado, de acordo com o parecer no 113.551-02/10/2012 deste órgão.

\section{RESULTADOS}

A amostra foi constituída por 110 idosas, com idade entre 60 e 82 anos (média de 67 anos). As características sociodemográficas estão descritas na Tabela 1. Observa-se que as pessoas na faixa etária de 60-70 anos correspondem à maioria das entrevistadas $(70,9 \%)$. 
Tabela 1. Características sociodemográficas e socioeconômicas de idosas participantes de grupo de convivência do município Bocaiuva-MG/Brasil, 2012 ( $n=110)$

\begin{tabular}{|c|c|c|}
\hline Variáveis & $\mathbf{n}$ & $\%$ \\
\hline \multicolumn{3}{|l|}{ Faixa Etária } \\
\hline 60 a 70 anos & 78 & 70,9 \\
\hline 71 a 80 anos & 30 & 27,2 \\
\hline 81 anos ou mais & 2 & 1,8 \\
\hline \multicolumn{3}{|l|}{ Estado civil } \\
\hline Com companheiro & 43 & 39,1 \\
\hline Sem companheiro & 67 & 60,9 \\
\hline \multicolumn{3}{|l|}{ Escolaridade } \\
\hline Analfabeto & 28 & 25,5 \\
\hline 1 a 8 anos de escolaridade & 67 & 60,9 \\
\hline 9 ou mais anos de escolaridade & 24 & 21,7 \\
\hline \multicolumn{3}{|l|}{ Renda familiar } \\
\hline Até um salário mínimo & 29 & 26,4 \\
\hline Dois ou mais salários mínimos & 81 & 73,6 \\
\hline \multicolumn{3}{|l|}{ Arranjo familiar } \\
\hline Sozinha & 22 & 20 \\
\hline Esposo & 15 & 13,6 \\
\hline Filhos(as) & 31 & 28,8 \\
\hline Esposo, filhos(as) e/ou outros & 42 & 37,6 \\
\hline \multicolumn{3}{|l|}{ Religião } \\
\hline Católica & 79 & 71,8 \\
\hline Outras & 31 & 28,2 \\
\hline \multicolumn{3}{|l|}{ Recebem aposentadoria } \\
\hline $\operatorname{Sim}$ & 82 & 74,5 \\
\hline Não & 28 & 25,5 \\
\hline \multicolumn{3}{|l|}{ Acesso a planos de saúde } \\
\hline Sim & 16 & 14,5 \\
\hline Não & 94 & 85,5 \\
\hline
\end{tabular}

Elaborado pelos autores (2020)

Na Tabela 2, são descritas as médias atingidas pelas idosas em cada questão, agrupadas por faceta do WHOQOL-OLD. 
Tabela 2. Descrição das questões de cada faceta e médias do questionário WHOQOL-OLD em 110 idosas que frequentavam o grupo de convivência da Cidade de Bocaiuva-MG em 2012.

\begin{tabular}{|c|c|c|}
\hline Faceta/Questão & Média & $\begin{array}{l}\text { Desvio- } \\
\text { padrão }\end{array}$ \\
\hline Funcionamento do Sensório (FS) & 9,85 & 2,645 \\
\hline Old 01 - Impacto da perda dos sentidos na vida diária & 2,45 & 1,037 \\
\hline Old 02 - Perda dos sentidos afeta participação nas atividades & 2,00 & 1,005 \\
\hline Old 10 - Funcionamento dos sentidos afeta habilidade de interação & 1,75 & 0,981 \\
\hline Old 20 - Como avalia os sentidos & 3,65 & 0,933 \\
\hline Autonomia (AUT) & 14,57 & 2,011 \\
\hline Old 03 - Liberdade para tomar as decisões & 3,72 & 0,803 \\
\hline Old 04 - Sente que controla o próprio futuro & 3,56 & 0,736 \\
\hline Old 05 - Pessoas ao redor respeitam a sua liberdade & 3,85 & 0,593 \\
\hline Old 11 - Consegue fazer as coisas que gostaria & 3,45 & 0,785 \\
\hline Atividades Passadas, Presentes e Futuras (PPF) & 15,1 & 1,774 \\
\hline Old 12 - Satisfação com oportunidades para realizações na vida & 3,60 & 0,624 \\
\hline Old 13 - Recebeu o reconhecimento que merece na vida & 3,65 & 0,722 \\
\hline Old 15 - Satisfação com aquilo que alcançou na vida & 3,98 & 0,606 \\
\hline Old 19 - Quão feliz com coisas a esperar daqui pra frente & 3,86 & 0,656 \\
\hline Participação Social (PSO) & 15,6 & 1,412 \\
\hline Old 14 - Tem o suficiente para fazer em cada dia & 3,68 & 0,541 \\
\hline Old 16 - Satisfação com a maneira com a qual usa seu tempo & 3,97 & 0,533 \\
\hline Old 17 - Satisfação com o nível de atividades & 3,92 & 0,623 \\
\hline $\begin{array}{l}\text { Old } 18 \text { - Satisfação com oportunidades de participar de atividades } \\
\text { na comunidade }\end{array}$ & 4,07 & 0,631 \\
\hline Morte e Morrer (MEM) & 8,66 & 3,226 \\
\hline Old 06 - Preocupação com a maneira que irá morrer & 2,05 & 1,233 \\
\hline Old 07 - Medo de não poder controlar a morte & 1,85 & 1,262 \\
\hline Old 08 - Medo de morrer & 1,49 & 0,965 \\
\hline Old 09 - Teme sofrer dor antes de morrer & 3,26 & 1,217 \\
\hline Intimidade (INT) & 13,51 & 2,294 \\
\hline Old 21 - Sentimento de companheirismo na vida & 2,53 & 1,406 \\
\hline Old 22 - Sentimento de amor em sua vida & 3,65 & 0,808 \\
\hline Old 23 - Oportunidade para amar & 3,73 & 0,703 \\
\hline Old 24 - Oportunidade para ser amado & 3,61 & 0,755 \\
\hline
\end{tabular}

Elaborado pelos autores (2020)

A faceta que apresentou maior escore foi Participação Social (PSO), e com menor pontuação, a faceta Morte e Morrer (MEM). 
$\mathrm{Na}$ Tabela 3, foram descritas as médias atingidas pelas idosas em cada domínio do WHOQOL-bref. O domínio psicológico foi o que apresentou maior escore.

Tabela 3. Média e desvio-padrão de cada domínio do WHOQol-bref.

\begin{tabular}{lll}
\hline Domínios & Média & Desvio-padrão \\
\hline Domínio Físico & 66,87 & 14,68 \\
Domínio Psicológico & 67,45 & 9,86 \\
Domínio Social & 61,68 & 13,70 \\
Domínio Ambiental & 63,65 & 10,85 \\
Domínio geral sobre qualidade de vida & 67,84 & 18,11 \\
\hline
\end{tabular}

Elaborado pelos autores (2020)

A Tabela 4 apresenta as médias em cada domínio e faceta da qualidade de vida das idosas, entre aquelas que possuem e não possuem um companheiro. Com base nos resultados da Razão de Prevalência (RP) confirmado pelo teste não-paramétrico de Mann-Whitney, é possível observar que existe diferença significante entre quem tem e não tem companheiro com relação à faceta Intimidade, ao domínio social, e à qualidade de vida geral.

Tabela 4. Comparação da qualidade de vida entre idosas que possuem e não possuem companheiro

\begin{tabular}{lcccccc}
\hline & \multicolumn{2}{c}{ Com companheiro } & \multicolumn{2}{c}{ Sem companheiro } & p-valor & RP \\
\cline { 2 - 5 } & Média & Desvio-padrão & Média & Desvio-padrão & & \\
\cline { 2 - 5 } Funcionamento do & 9,26 & 2,83 & 10,22 & 2,47 & 0,103 & 0,9 \\
Sensório & 14,70 & 1,78 & 14,49 & 2,16 & 0,892 & 1,0 \\
Autonomia & 8,51 & 3,31 & 8,76 & 3,20 & 0,600 & 1,0 \\
Morte e Morrer & & & & & & \\
Atividades passadas, & 15,30 & 1,44 & 14,96 & 1,96 & 0,221 & 1,0 \\
presentes e futuras & 15,70 & 1,12 & 15,61 & 1,58 & 0,731 & 1,0 \\
Participação social & 14,53 & 2,32 & 12,85 & 2,03 & $<0,001^{*}$ & 1,1 \\
Intimidade & 24,19 & 2,11 & 23,55 & 2,33 & 0,119 & 1,0 \\
Domínio Físico & 21,09 & 2,20 & 20,64 & 2,02 & 0,382 & 1,0 \\
Domínio Psicológico & 10,93 & 1,55 & 10,06 & 1,62 & $0,007^{*}$ & 1,1 \\
Domínio Social & 28,77 & 3,62 & 27,82 & 3,37 & 0,219 & 1,0 \\
Domínio Ambiente & 7,88 & 1,24 & 7,19 & 1,46 & $0,017^{*}$ & 1,1 \\
QV geral & & & & &
\end{tabular}

Teste não-paramétrico de Mann-Whitney

Interpretação: $R P=1,0$ sem associação; $R P>1,0$ Associação Positiva; $R P<1,0$ Associação negativa.

Elaborado pelos autores (2020) 


\section{DISCUSSÃO}

O processo de envelhecimento populacional, observado em todos os continentes do mundo, vem marcando profundamente as sociedades atuais. O aumento do número de idosos está a impor mudanças profundas nos modos de pensar e viver a velhice na sociedade. Todos os segmentos da vida humana já estão sendo desafiadas nesse sentido: é observado que, de um lado, já há maior preocupação por parte dos governos em assumir políticas favoráveis à manutenção da autonomia e independência das pessoas idosas; de outro lado, as próprias pessoas idosas, que têm buscado maior engajamento social, principalmente nos países onde a democracia e o Estado de direito são conquistas efetivas da sociedade ${ }^{17}$.

O maior acesso aos serviços de saúde, bem como aos bens sociais como educação e renda, tem possibilitado um olhar diferenciado e associado da maneira como se vê a própria imagem do abandono associada à velhice. Atitudes como políticas previdenciárias e de assistência social, em conjunto com a expansão e qualificação da estratégia saúde da família têm contribuído para horizontes mais positivos na vida dos brasileiros com 60 anos e mais ${ }^{17}$.

Quando são avaliadas as diferentes facetas do questionário WHOQOL-OLD aqui aplicado verifica-se que contribuem para a qualidade de vida com impactos diferentes. A faceta Participação Social (PSO) foi a que mais contribuiu entre as idosas pesquisadas. Semelhante resultado foi encontrado em estudo de Santos et al. ${ }^{18} \mathrm{com}$ participantes de ambos os sexos. A respeito da importância da participação de pessoas idosas em grupos de convivência, estudos pontuam que os grupos possibilitam, de alguma forma, melhorar aspectos físicos e psicossociais do idoso, uma vez que estimula a adesão dessas pessoas a hábitos saudáveis, à pratica regular exercícios físicos e ao exercício da participação social ${ }^{19-20}$. Participar de grupos direcionado à promoção da saúde proporciona melhorias de diversos aspectos da QV dos idosos, sobretudo dos relacionados à participação social e à construção/manutenção de relacionamento interpessoal ${ }^{18}$.

A faceta Atividades Passadas, Presentes e Futuras (PPF) foi uma das que também mais contribuiu na qualidade de vida das idosas estudadas. Essa dimensão avalia a satisfação do idoso pelas conquistas e reconhecimento obtidos na vida e as perspectivas futuras. Em estudo que comparou a qualidade de vida de idosos jovens e muito idosos, essa faceta também contribuiu com alto valor do escore, indicando que a qualidade de vida de idosos é influenciada pela oportunidade de amar e sentir-se amado, estar satisfeito com suas realizações, objetivos alcançados e projetos durante a vida ${ }^{21}$.

Os projetos futuros propiciam aos idosos a identificação do sentido das suas vidas, reforçando a autoestima, a funcionalidade e a capacidade de vislumbrar ainda outras novas aspirações. Dessa forma, é inegável que os grupos relativos à terceira idade ne- 
cessitem trabalhar a potencialidade do ser humano e sua capacidade de criar projetos e concretizá-los ${ }^{13}$.

A faceta autonomia, em que se avalia a independência na velhice e a capacidade da pessoa em estabelecer as suas regras de condutas e segui-las, correspondeu ao terceiro escore mais elevado neste estudo, o que indica que as idosas avaliaram positivamente esse domínio, e, são, portanto, pessoas que conseguem fazer as coisas de que gostariam e possuem liberdade para tomar suas decisões. Vale ressaltar que a promoção da autonomia é um dos propósitos da Política Nacional de Saúde da Pessoa Idosa (PNSPI) 17. Interessante ainda é que os grupos de convivência, tais como o aqui observado, são uma importante forma de interação, inclusão social e uma maneira de promover a autonomia, de viver com dignidade e dentro do âmbito de ser e estar saudável ${ }^{22}$. Os grupos de convivência possibilitam ao indivíduo adquirir maior autonomia, melhorar sua qualidade de vida, senso de humor, e inclusão social. Este último fator influencia sobremaneira na continuidade dos idosos nos programas e nas mudanças positivas alcançadas em suas vidas ${ }^{23}$.

A faceta intimidade, com escore seguido da dimensão "Autonomia" avaliou a capacidade de as idosas terem relações pessoais e íntimas. Deve-se destacar, que, embora, mais da metade $(60,9 \%)$ das participantes não tivessem cônjuge, estavam inseridas em um arranjo familiar do tipo "com filhos(as) ou esposo, filhos(as) e/ou outros", o que permite inferir que o suporte familiar interfere positivamente nesse domínio. Esse resultado diverge do estudo realizado por Santos e Cunha (2014) ${ }^{24}$ em que essa faceta se apresentou com uma das menores pontuações atingidas.

A faceta "Função Sensorial" avalia o funcionamento sensorial (visão, audição, tato, olfato e paladar) e o impacto da perda das habilidades sensoriais na qualidade de vida. No WHOQOL-OLD esse domínio foi um dos que apresentaram menor pontuação. Este valor indica que, nesta faceta, as idosas apresentam sua qualidade de vida comprometida. As interações sociais da pessoa idosa podem ser influenciadas pelas limitações provenientes da redução do funcionamento sensorial, as quais devem ser prontamente identificadas pelos profissionais de saúde e diferenciadas das alterações associadas a doenças ${ }^{25}$.

Um achado instigante neste estudo é que, embora, as facetas participação social e atividades passadas, presentes e futuras tenham sido as de maior pontuação, refletindo uma avalição positiva da qualidade de vida das idosas nesses domínios, quando se remete à faceta morte e morrer, em que se é possível avaliar a preocupação com a maneira que se irá morrer, o medo de não poder controlar a morte, o medo de morrer e o temor de sofrer antes de morrer, percebe-se que a média do escore alcançou o menor valor $(8,6)$ com desvio padrão de 4,6 . Essa pontuação indica que as idosas pesquisadas, 
mesmo tendo avaliado de forma satisfatória a maneira com a qual usa seu tempo e tendo recebido o reconhecimento que merece na vida, não têm controle de preocupações e temores sobre a morte e o morrer, e possivelmente não estão preparadas para morrer.

Diferentemente deste estudo, em estudo que objetivou avaliar a qualidade de vida de mulheres idosas no que diz respeito à faceta morte e morrer do WHOQOL-OLD observou-se para esse domínio a maior pontuação ${ }^{18,26}$. Para o futuro seria interessante investigar quais fatores, sejam de natureza cultural ou não, estariam influenciando mais a concepção do morrer entre os idosos.

A participação em atividades do cotidiano, sobretudo na comunidade, e a manutenção da autonomia influenciaram positivamente na $\mathrm{QV}$, possivelmente por sentir-se útil para a sociedade, ser ouvido e ter suas opiniões respeitadas. O combate com a morte com o passar dos anos e a perda de parentes e amigos pode explicar a baixa pontuação no domínio morte e morrer. Acrescenta-se que valores elevados de escore nesse domínio demonstram que aceitar a finitude da vida pode resultar em paz interior e, consequentemente, contribuir para maior tempo de vida com qualidade ${ }^{21}$.

Ao se analisar a contribuição dos diferentes domínios do questionário WHOQOL-BREF, o domínio que mais contribuiu foi o meio ambiente, seguido do psicológico e relações sociais com o mesmo percentual e por último o físico.

Quanto ao menor escore no domínio meio ambiente, que avalia segurança física e proteção, participação em, e oportunidades de recreação/lazer, recursos financeiros, transporte, dentre outros, pôde-se inferir que as idosas pesquisadas sentem-se seguras e protegidas, e que maiores oportunidades de atividades de lazer e recreação podem ter contribuído para o impacto na QV, avaliado por este domínio.

Esse resultado é diferente dos dois estudos com idosos em grupo de convivência que utilizaram o instrumento WHOQOL-BREF. Em um estudo realizado em uma Unidade Básica de Saúde no município de São Paulo ${ }^{24}$, esse domínio obteve o menor escore, e, em outra pesquisa realizada na Universidade Católica do Rio Grande do Sul ${ }^{13}$, o domínio que mais contribuiu na qualidade de vida dos idosos foi o social, seguido do ambiental, psicológico e físico. Ressalta-se a questão do gênero neste estudo pode ter interferido nos resultados, uma vez que, diferentemente, dos dois estudos, este foi constituído somente por pessoas do sexo feminino, assim, sugere-se que outros estudos semelhantes a este para melhor comparação da qualidade de vida de mulheres idosas em grupo de convivência.

Outro achado importante nesta pesquisa foi à observação de que há diferença significativa entre quem tem e não tem companheiro com relação à faceta Intimidade, ao domínio social, e à qualidade de vida geral. Isso reflete como a presença do outro e a cumplicidade com o mesmo são potenciais geradores de saúde. Se a formação essen- 
cialmente humana se dá pelo contato e interação com as pessoas, na velhice, essa interação tem um impacto ainda maior, com repercussões nem sempre imagináveis.

Apesar de não interferir no mérito da pesquisa, uma limitação deste estudo foi refletir a realidade de um dado local, em uma dada região brasileira. Não se sabe até que ponto esses resultados podem ser extrapolados à realidade do Brasil como um todo. Ainda assim, poucos trabalhos envolveram a aplicação de instrumentos tão importantes para acessar a qualidade de vida em idosos. É importante salientar também que apenas idosas participantes do grupo de convivência foram incluídas no estudo, não permitindo assim generalizar os resultados encontrados. Recomenda-se a realização de estudos que incluam os idosos do sexo masculino bem como aqueles não participantes de grupos de convivência, no intuito de estabelecer melhor o perfil da qualidade de vida entre pessoas idosas. Ainda, também os autores sugerem relacionar as variáveis de qualidade de vida com determinantes sociais e de saúde.

\section{CONCLUSÃO}

$\mathrm{Na}$ análise dos dados do WHOQOL-OLD aplicado na população envolvida, a percepção que menos contribuiu para a qualidade de vida foi a relacionada à Morte e Morrer (MEM). Esse dado pode significar que as pessoas não estão se preocupando tanto com a forma como irão morrer, ou tão pouco se prendem a preocupações com o porvir. De outra forma, pode também significar que, para essas idosas, o pensar na morte ainda é uma tarefa difícil, a qual se pretende evitar. Há que se refletir aqui, que interferências culturais locais podem alterar de forma importante o significado da morte para as pessoas. O domínio social (PSO) apresentou o maior escore dentre os domínios da qualidade de vida. As atividades desenvolvidas nos grupos de convivência proporcionam momentos de trocas de informação, atividades culturais, religiosas e de lazer e parecem favorecer a socialização das participantes, contribuindo, significativamente para a autonomia e qualidade de vida das idosas.

No WHOQOL-Bref a percepção mais negativa de qualidade de vida foi o domínio físico. Funcionalmente, as pessoas se consideram mais debilitadas, tornando necessário as práticas de melhoria da qualidade de vida no que diz respeito a sua capacidade funcional. O bem-estar do idoso é resultado do equilíbrio entre as diversas dimensões da capacidade funcional, não significando ausência de problemas em todas as dimensões, mas podendo ser atingido, independente da presença ou não de enfermidades. $O$ encontrado reflete justamente como o equilíbrio dessas dimensões exerce impacto no bem-estar dos idosos.

Destaca-se a escassez de trabalhos utilizando ambos, o WHOQOL-OLD e BREF, no contexto de grupos operativos, dificultando o estabelecimento de comparações e a 
implementação de medidas. Foram encontrados somente dois estudos que utilizaram o WHOQOL-OLD conjuntamente com o WHOQOL- BREF em grupos de convivência. Assim, o presente estudo é válido por reconhecer a importância da aplicação desses dois instrumentos, no âmbito da estratégia de grupo, para idosos, com objetivo de se avaliar a qualidade de vida.

A qualidade de vida é um importante indicador de saúde e os questionários WHOQOL-OLD e BREF são instrumentos preciosos que podem avaliá-la, e ainda dar subsídios para tomadas de ações que visem promover o bem-estar da população idosa.

\section{REFERÊNCIAS}

1. Closs VE, Schwanke CHA. A evolução do índice de envelhecimento no Brasil, nas suas regiões e unidades federativas no período de 1970 a 2010. Rev. bras. geriatr. gerontol. [Internet]. 2012 [acesso em 2020 dez 21];15(3):443-458. Disponível em: https://doi.org/10.1590/S1809-98232012000300006.

2. Guimarães M. Para uma (re) Educação dos Idosos do Grupo "PROGRAMA DA MELHOR IDADE" de Paraíso/Tocantins, Frente às Mudanças do Mundo Contemporâneo. Dissertação de mestrado, 2011. Disponível em: http://recil.grupolusofona.pt/bitstream/10437/1531/1/Disserta\%C3\%A7\%C3\%A30\%20-\%20 Marisa\%20Guimar\%C3\%A3es.pdf.

3. Brasil. Ministério da Saúde. Atenção à saúde da pessoa idosa e envelhecimento. Secretaria de Atenção à Saúde, Departamento de Ações Programáticas e Estratégicas, Área Técnica Saúde do Idoso. - Brasília, 2006. Disponível em: https://bvsms.saude.gov.br/bvs/publicacoes/atencao_saude_pessoa_idosa_enveIhecimento_v12.pdf

4. Lima LCV, Bueno CMLB. Envelhecimento e Gênero: a Vulnerabilidade de Idosas no Brasil. Revista Saúde e Pesquisa 2009; 2(2): 273-280. Disponível em: https://periodicos.unicesumar.edu.br/index.php/ saudpesq/article/view/1173/792

5. Castro JA de; Aquino L (Orgs.). Juventude e políticas sociais no Brasil.Brasília: Ipea, abril de 2008 (Texto para discussão, 1.335). Disponível em: http:http://www.abep.nepo.unicamp.br/docs/outraspub/cairo15/ Cairo15_4camaranomellokanso.pdf

6. Coelho DNP, Daher DV, Santana RF, Espírito Santo FH. Percepção de Mulheres Idosas sobre Sexualidade: Implicações de gênero e no cuidado de enfermagem. Revista Rene. 2010; 11(4):163-173. Disponível em: http://www.periodicos.ufc.br/rene/article/view/4641/3466.

7. Sprangers MA, Regt EB, Andries F, van Agt HME, Bijl RV, de Boer JB, et al. Which chronic conditions are associated with better or poorer quality of life? J Clin Epidemiol. 2000;53(9):895-907. Disponível em: http:// dx.doi.org/10.1016/s0895-4356(00)00204-3

8. Rocha SV, Santos CA, Souza NR, Carneiro LRV. Qualidade de vida entre mulheres participantes de grupos de convivência. Revista APS: Juiz de Fora. 2010;13(3):352-356. Disponível em: http://www.aps.ufff. br/index.php/aps/article/download/773/347

9. WHOQOL Group. The World Health Organization Quality of Life assessment (WHOQOL): position paper from the World Health Organization. Soc Sci Med. 1995 Nov;41(10):1403-9. Disponível em: http://dx.doi. org/10.1016/0277-9536(95)00112-k

10. Miranda LC, Banhato EFC. Qualidade de vida na terceira idade: a influência da participação em grupos. Psicologia em Pesquisa 2008; 2(01): 69-80. Disponível em: https://doi.org/10.24879/200800200100420

11. Power M, Schmidt S. Manual WHOQOL-OLD. Genebra: 5. World Health Organization; 1998. p.19 p.]. Disponível em: http://www.ufrgs.br/psiq/WHOQOL-OLD\%20Manual\%20POrtugues.pdf

12. Power M, Quinn K, Schmidt S; WHOQOL-OLD Group. Development of the WHOQOL-old module. Qual Life Res. 2005;14(10):2197-214. Disponível em: http://dx.doi.org/10.1007/s11136-005-7380-9 
13. Serbim, AK, Figueiredo, AEPL. Qualidade de vida de idosos em um grupo de convivência. Scientia Medica. 2011; 21(4):166-172, 2011. Disponível em: http//revistaseletronicas.pucrs.br/ojs/index.php/scientiamedica/.../7237

14. Silva MIS, Kinoshita F. A participação dos idosos nos grupos de convivência como uma possibilidade de um envelhecimento saudável. Extensio, Revista Eletrônica de Extensão. 2009; 6(7). Disponível em: https://periodicos.ufsc.br/index.php/extensio/article/view/1807-0221.2009v6n7p133/10437

15. Fleck MP, Chachamovich E, Trentini C. Development and validation of the Portuguese version of the WHOQOL-OLD module. Rev. Saúde Pública [Internet]. 2006 [acesso em 2020 dez 21]; 40(5): 785-791. Disponível em: https://doi.org/10.1590/S0034-89102006000600007

16. Fleck MPA. O instrumento de avaliação de qualidade de vida da Organização Mundial da Saúde (WHOQOL-100): características e perspectivas. Ciênc. saúde coletiva [Internet]. 2000 [acesso em 2020 dez 21] 5(1):33-38. Disponível em: http://dx.doi.org/10.1590/S1413-81232000000100004

17. Brasil. Ministério da Saúde. Envelhecimento e saúde da pessoa idosa. 19 ed. Secretaria de Atenção à Saúde. Brasília: Ministério da Saúde, 2006b.192 p. Disponível em: http://bvsms.saude.gov.br/bvs/publicacoes/atencao_saude_pessoa_idosa_envelhecimento_v12.pdf

18. Santos LF, Oliveira LMAC, Barbosa MA, Nunes DP, Brasil, VV. "Qualidade de vida de idosos que participam de grupo de promoção da saúde." Enfermería Global. 2015; 40:(12). Disponível em: http://scielo. isciii.es/pdf/eg/v14n40/pt_clinica1.pdf

19.Nogueira ALG, Munari DB, Santos LF, Oliveira LMAC, Fortuna CM. Fatores terapêuticos identificados em um grupo de Promoção da Saúde de Idosos. Rev. esc. enferm. USP [Internet]. 2013 [acesso em 2020 dec 21]; 47(6): 1352-1358. Disponível em: http://dx.doi.org/10.1590/S0080-623420130000600015

20. Benedetti TRB, Mazo GZ, Borges LJ. Condições de saúde e nível de atividade física em idosos participantes e não participantes de grupos de convivência de Florianópolis. Cien Saude Colet. 2012;17(8):208793.

21. Maués $C R$, Paschoal SMP, Jaluul $O$, França CC, Jacob Filho W. Avaliação da qualidade de vida: comparação entre idosos jovens e muito idosos. Rev Bras Clin Med. 2010; 8:405-10. Disponível em: http:// files.bvs.br/upload/S/1679-1010/2010/v8n5/007.pdf

22. Wichmann FMA, Couto NA, Areosa SVC, Montañés MCM. Grupos de convivência como suporte ao idoso na melhoria da saúde. Rev. bras. geriatr. gerontol. [Internet]. 2013 [acesso em 2020 dez 06];16(4): 821-832. Disponível em: http://dx.doi.org/10.1590/S1809-98232013000400016.

23. Almeida EA, Madeira GD, Arantes PMM, Alencar MA. Comparação da qualidade de vida entre idosos que participam e idosos que não participam de grupos de convivência na cidade de Itabira-MG. Rev Bras Geriatr Gerontol [Internet] 2010 [acesso em 11 ago 2020];13(3):435-44. Disponível em: https://doi. org/10.1590/S1809-98232010000300010

24. Santos GS, Cunha IC. Avaliação da qualidade de vida de mulheres idosas na comunidade. R. Enferm. Cent. O. Min. 2014; 4:1135-1145. Disponível em: https://doi.org/10.19175/recom.v0i0.593

25. Tavares DMS, Gomes NC, Dias FA, Santos NMF. Fatores associados à qualidade de vida de idosos com osteoporose residentes na zona rural. Esc Anna Nery. 2012; 16:371-8. Disponível em: https://doi. org/10.1590/S1414-81452012000200023

26.Carvalho ED, Valadares ALR, Costa-Paiva LH, Pedro AO, Morais SS, Pinto-Neto AM. Atividade física e qualidade de vida em mulheres com 60 anos ou mais: fatores associados. Rev. Bras. Ginecol. Obstet. 2010;32(9):433-440. Disponível em https://www.scielo.br/pdf/rbgo/v32n9/v32n9a04.pdf 